\title{
An Optimal Feedback Regulation of Nonlinear Singularly Perturbed Systems via Slow Manifold Approach*
}

\author{
KHASHAYAR KHORASANI $\dagger$
}

Key Words-Singular perturbations; optimal control; nonlinear systems; model reduction.

\begin{abstract}
A concept of exact slow optimal control is defined for a general class of nonlinear singularly perturbed systems utilizing the slow manifold theory. Under a set of conditions an exact optimal feedback regulation restricted to the slow manifold is obtained. The result is applied to a class of nonlinear systems with nonlinear fast actuators. It is shown that by adding an extra compensating slow control to the near optimal control an exact optimal feedback regulation is achieved on the manifold. An upper bound on the perturbation parameter is obtained under which the result is valid.
\end{abstract}

\section{Introduction}

To AVOID the ill conditioning and dimensionality problems, standard procedure in the literature (Chow and Kokotovic, 1976, 1978a, b, 1981) for regulation (stabilization) of nonlinear singularly perturbed systems is to define two lower order subproblems, the reduced slow and fast subproblems. Solution of the optimal control for each subproblem is then used to define a composite control. It can be shown that the full regulator problem is well-posed with respect to parasitic parameters in the sense that as the perturbation parameter $\varepsilon$ tends to zero the cost of the full problem tends to the cost of the reduced slow subproblem. For sufficiently "small" perturbation values this near-optimality property can be satisfactory. In this paper we present an approach to improve upon this approximate result. The contribution of this paper is twofold. First, for a general class of nonlinear singularly perturbed systems an exact optimal control strategy is achieved by utilizing the slow manifold theory (Fenichel, 1979; Hoppensteadt, 1971). This is accomplished by defining an exact slow subproblem. The solution to this problem is called an exact slow optimal control. In contrast to the near optimal result this result is valid for all possible values of the perturbation parameter and not just at $\varepsilon=0$. Second, for a class of nonlinear systems which are linear in the fast variable and control, provided the system satisfies a rank condition an extra compensating control $u_{1}$ exists such that the slow control $u=u_{0}+\varepsilon u_{1}$ is an exact slow optimal control strategy for the exact slow subproblem, where $u_{0}$ is an optimal control for the reduced slow subproblem.

2. Slow manifold theory for singularly perturbed systems Consider the nonlinear singularly perturbed system

$$
\begin{gathered}
\dot{x}=f(x, z, u, \varepsilon), \quad x \in R^{n} \\
\varepsilon \dot{z}=g(x, z, u, \varepsilon), \quad z \in R^{m}, \quad u \in R^{r} \quad \varepsilon>0 .
\end{gathered}
$$

"Received 1 January 1986; revised 15 October 1986; revised 19 June 1987 . The original version of this paper was not presented at any IFAC meeting. This paper was recommended for publication in revised form by Associate Editor M. Jamshidi under the direction of Editor A. P. Sage.

† Department of Electrical and Computer Engineering, The University of Michigan-Dearborn, Dearborn, MI 48128, U.S.A.
In the $(n+m)$-dimensional state space of $x$ and $z$, an $n$-dimensional manifold $M_{e}$, depending on the scalar parameter $\varepsilon$, can be defined by an expression of explicit dependence of $z$ on $x, u$ and $\varepsilon$, namely,

$$
M_{\varepsilon}: z=\Phi(x, u, \varepsilon) ; \quad x \in R^{n}, \quad z \in R^{m},
$$

where it is assumed that $\Phi$ is a sufficiently many times continuousiy differentiable function of $x, u$ and $\varepsilon$. For $M_{\varepsilon}$ to be an invariant manifold of (1)-(2), expression (3) must hold for all $t>t^{*}$ if it holds at $t=t^{*}$, that is,

$$
z\left(t^{*}, \varepsilon\right)=\Phi\left(x\left(t^{*}, \varepsilon\right), u, \varepsilon\right) \Rightarrow z(t, \varepsilon)=\Phi(x(t, \varepsilon), u, \varepsilon),
$$

for all $t \geq t^{*}$

Such an invariant manifold exists provided the Jacobian matrix $(\partial g / \partial z)_{z=0}$ is nonsingular for all $x$ and $u$ of interest. Differentiating (3) and using (1)-(2), $\Phi(x, u, \varepsilon)$ is determined by the so-called manifold condition

$$
\varepsilon \frac{\partial \Phi}{\partial x} f(x, \Phi(x, u, \varepsilon), u, \varepsilon)+\varepsilon \frac{\partial \Phi}{\partial u} \dot{u}=g(x, \Phi(x, u, \varepsilon), u, \varepsilon) .
$$

$\Phi(x, u, \varepsilon)$ must satisfy this partial differential equation for all $x$ of interest, and for all $\varepsilon \in\left(0, \varepsilon^{*}\right], \varepsilon^{*}>0$. The dynamics of system (1)-(2) restricted to this manifold are described by what we call the reduced exact slow subsystem

$$
\dot{x}=f(x, \Phi(x, u, \varepsilon), u, \varepsilon), \quad x \in R^{n} .
$$

The solution to $\Phi(x, u, \varepsilon)$ is sought by expressing $f, g, u$ and the manifold in a power series about $\varepsilon=0$ in (4) and equating terms with like powers in $\varepsilon$. That is, we let

$$
\begin{gathered}
u(x)=u_{0}(x)+\varepsilon u_{1}(x)+O\left(\varepsilon_{2}\right), \\
\Phi(x, u, \varepsilon)=\varphi_{0}\left(x, u_{0}\right)+\varepsilon \varphi_{1}(x, u)+O\left(\varepsilon^{2}\right) .
\end{gathered}
$$

Substituting (6)-(7) into (4), explicit solutions for $\varphi_{0}, \varphi_{1}$, etc. can be obtained (Kokotovic and Khorasani, 1984). When we set $\varepsilon=0$, an "uncorrected" slow manifold $M_{0}$ is defined by $z=\varphi_{0}\left(x, u_{0}\right)$, such that $g\left(x, \varphi_{0}\left(x, u_{0}\right), u_{0}, 0\right)=0$. The uncorrected slow subsystem is now given by the restriction of (1)-(2) to $M_{0}$, that is,

$$
\dot{x}=f\left(x, \varphi_{0}\left(x, u_{0}(x)\right), u_{0}(x), 0\right) .
$$

At this point it should be remarked that system (5), in contrast to the uncorrected slow subsystem, does not represent an approximation to (1)-(2). Fortunately, the uncorrected slow subsystem can be improved. From the manifold condition it is easily seen that $\varphi_{1}(x, u)$ is

$$
\begin{array}{r}
\varphi_{1}(x, u)=\left(\frac{\partial g}{\partial z}\right)_{z=\varphi_{0}}^{-1}\left[\left(\frac{\partial \varphi_{0}}{\partial x}+\frac{\partial \varphi_{0}}{\partial u} \frac{\partial u_{0}}{\partial x}\right) f\left(x, \varphi_{0}, u_{0}, 0\right)\right. \\
\left.-\frac{\partial g}{\partial u} u_{1}-\left(\frac{\partial g}{\partial \varepsilon}\right)_{z=\varphi_{0}}\right] .
\end{array}
$$


With the "corrected" slow manifold $M_{1}$ defined by $z=\varphi_{0}+\varepsilon \varphi_{1}$, which is an $O(\varepsilon)$ closer to the exact manifold $M_{\varepsilon}$ compared to the uncorrected manifold $M_{0}$, the first-order-corrected slow subsystem is then given by

$$
\dot{x}=f\left(x, \varphi_{0}\left(x, u_{0}(x)\right)+\varepsilon \varphi_{1}\left(x, u_{0}+\varepsilon u_{1}\right), u_{0}+\varepsilon u_{1}, \varepsilon\right) .
$$

This process can be continued until the desired accuracy is achieved. With the above results in hand we present our first result in the next section.

\section{Nonlinear singularly perturbed regulator problem}

The problem in this section is to optimally control the nonlinear system

$$
\begin{aligned}
\dot{x} & =f(x, z, u, \varepsilon), \quad x \in R^{n} \\
\varepsilon \dot{z} & =g(x, z, u, \varepsilon), \quad z \in R^{m}, \quad u \in R^{r}
\end{aligned}
$$

with respect to the performance index

$$
J=\int_{0}^{\infty} L(x, z, u) \mathrm{d} t,
$$

where we assume that the scalar function $L$ is positive definite with respect to its arguments except at $x=z=u=0$ where it is zero, and $f$ and $g$ are continuously many times differentiable in the domain of interest.

The approach to the full problem is to treat $\varepsilon$ as a nonzero constant parameter and assume that a differentiable optimal value function $V(x, z, \varepsilon)$ exists which satisfies the optimality principle

$$
0=\min _{u}\left[L(x, z, u)+\frac{\partial V}{\partial x} f(x, z, u, \varepsilon)+\frac{1}{\varepsilon} \frac{\partial V}{\partial z} g(x, z, u, \varepsilon)\right] .
$$

The above problem is difficult to solve, in general, due to high dimensionality and ill conditioning problems caused by the parasitic dynamics. The approach taken in Chow and Kokotovic (1978a, b, 1981) (see also Saberi and Khalil, 1985) is to define two lower order slow and fast subproblems, where solutions (if they exist) are much easier to obtain. The concept of composite control is then utilized to define an optimal control for the full system (9)-(11).

The reduced slow subproblem is to optimally control the uncorrected slow subsystem

$$
\dot{x}=f\left(x, \varphi_{0}\left(x, u_{0}\right), u_{0}, 0\right)
$$

with respect to the performance index

$$
J_{s}=\int_{0}^{\infty} L\left(x, \varphi_{0}\left(x, u_{0}\right), u_{0}\right) \mathrm{d} t .
$$

The approximation used in the subproblem modeling results in an approximate optimal control $u_{0}$. Our objective is to show that the slow manifold approach discussed in Section 1 would enable one to obtain an "exact optimal control" for the full problem at the reduced level modeling. To this end let the slow manifold of (9)-(10) be given by $z=\Phi(x, u, \varepsilon)$, and consequently, the reduced exact slow subsystem of (9) $-(10)$, by

$$
\dot{x}=f(x, \Phi(x, u, \varepsilon), u, \varepsilon) .
$$

The exact slow subproblem is now to optimally control the reduced exact slow subystem with respect to the performance index

$$
J_{s}=\int_{0}^{\infty} L\left(x, \varphi_{0}\left(x, u_{0}\right), u_{0}\right) \mathrm{d} t
$$

Lemma 3.1. Let $V_{e}$ be the exact optimal value function which satisfies the optimality principle

$$
0=\min _{u}\left[L(x, \Phi(x, u, \varepsilon), u)+\frac{\partial V_{e}}{\partial x} f(x, \Phi(x, u, \varepsilon), u, \varepsilon)\right]
$$

and which is sufficiently many times continuously differentiable with respect to $x$ in the domain of interest resulting in $u=\Psi\left(x, \frac{\partial V_{e}}{\partial x}, \varepsilon\right)$, and which satisfies the exact
Hamilton-Jacobi equation

$$
\begin{aligned}
& 0=L\left(x, \Phi\left(x, \Psi\left(x, \frac{\partial V_{e}}{\partial x}, \varepsilon\right), \varepsilon\right), \Psi\left(x, \frac{\partial V_{e}}{\partial x}, \varepsilon\right)\right) \\
& +\frac{\partial V_{e}}{\partial x} f\left(x, \Phi\left(x, \Psi\left(x, \frac{\partial V_{e}}{\partial x}, \varepsilon\right), \varepsilon\right), \Psi\left(x, \frac{\partial V_{e}}{\partial x}, \varepsilon\right), \varepsilon\right) .
\end{aligned}
$$

Then the optimal cost function of the reduced exact slow subsystem is equal to the optimal cost function of the full system restricted to the manifold $M_{\varepsilon}$.

Proof. (Outline.) It can be seen that the full problem Hamiltonian $H=L+\frac{\partial V}{\partial x} f+\frac{1}{\varepsilon} \frac{\partial V}{\partial z} g$ restricted to the manifold $M_{\varepsilon}$ using $\varepsilon \dot{\Phi}=g$ becomes $H=L+\left(\frac{\partial V}{\partial x}+\frac{\partial V}{\partial z} \frac{\partial \Phi}{\partial x}\right) f:=$ $L+\frac{\partial V_{e}}{\partial x} f$ from which (12) is established. The exact Hamilton-Jacobi equation follows along the same lines.

Definition. The control $u$ which satisfies (12) and (13) is said to be an exact slow optimal control for the full problem (9)-(10).

To illustrate the application of this result consider the following example. The problem is to optimally control the system

$$
\begin{aligned}
\dot{x} & =x z^{3} \\
\varepsilon \dot{z} & =z+6 \varepsilon x^{8}+u
\end{aligned}
$$

with respect to the performance index $J=\int_{0}^{\infty}\left(x^{8}+z^{4} / 2+\right.$ $\left.u^{4} / 2\right) \mathrm{d} t$. The uncorrected manifold $M_{0}($ at $\varepsilon=0)$ is given by $z=-u_{0}$. The reduced slow subproblem is to optimally control $\dot{x}=-x u_{0}^{3}$ with respect to $J_{s}=\int_{0}^{\infty}\left(x^{8}+u_{0}^{4}\right) \mathrm{d}$. From the optimality principle and the Hamilton-Jacobi equation we get $u_{0}=1.316 x^{2}$. Independent of this result, from the manifold condition we have $\Phi+6 \varepsilon x^{8}+u=\varepsilon(\partial \Phi / \partial x+$ $(\partial \Phi / \partial u)(\partial u / \partial x)) x \Phi^{3}$. A solution to the manifold $M_{\varepsilon}$ is chosen as $\Phi=-u$, which reduces the above equation to $(\partial u / \partial x) u^{3}=6 x^{7}$. A solution to this equation is given by $u=1.316 x^{2}$, which suggests that $\Phi=-1.316 x^{2}$, that is, $M_{\varepsilon}=M_{0}$. This means that the exact slow control which satisfies the manifold condition also satisfies the optimality conditions in this example. In other words, the exact manifold and slow control $u$ happen to be equal to the uncorrected manifold and slow control $u_{0}$, respectively. Therefore, the exact optimal value function is identical to the reduced optimal value function and, hence, $J_{e}=J_{s}$. The reduced fast subproblem is to optimally control

$$
\frac{\mathrm{d} \eta}{\mathrm{d} \tau}=\eta+u_{f}, \quad \tau=t / \varepsilon, \quad \eta=z+1.316 x^{2}
$$

with respect to the performance index $J_{f}=\int_{0}^{\infty}\left(\eta^{4} / 2+\right.$ $\left.u_{f}^{4} / 2\right) \mathrm{d} t$. The solution to this problem is easily obtained as $u_{f}=-1.445 \eta$. Lemma 3.1 states that the optimal cost function of the reduced exact slow subsystem is equal to the optimal cost function of the full system restricted to the manifoid $M_{\varepsilon}$. In other words, $J_{e}=\left.J\right|_{M_{\varepsilon}}$ with $z(0)=$ $-1.316 x^{2}(0)$ and $u=u_{0}$, and also we have $J^{\prime}=J_{e}$. However, for $z(0) \neq 1.316 x^{2}(0)$, the fast control $u_{f}$ is needed to stabilize the fast dynamics. After an exponential decay of the fast variable $z$ to $M_{\varepsilon}$, we have $J_{\varepsilon}=\left.J\right|_{M_{\varepsilon}}$ and $J=J_{e}+J_{f}+O(\varepsilon)$, where the $O(\varepsilon)$ term is due to the fast subproblem approximations. Moreover, there exists a time $t_{1}>0$ (after which the fast dynamics are evolving on the manifold), such that for all $t>t_{1}$ the cost to go of the full system is equal to that of the reduced exact slow subsystem.

\section{Regulator problem for systems with nonlinear fast} actuators

The results of the previous section are applied to a class of nonlinear systems with fast actuators (Chow and Kokotovic, 1978a, b, 1981). First, we compare our results with those of Chow and Kokotovic (1978a, b, 1981) and then present some new results for this class of systems. Generally speaking, we assume that there are two models: the true model and the 
reduced model. The true model contains unknown as well as parasitic parameters which result in a higher order model as compared to the reduced model. The reduced model obtained by neglecting parasitic parameters is used by the designer to derive an optimal control strategy for the regulator problem

$$
\dot{x}=a_{0}(x)+b_{0}(x) u_{0}
$$

subject to the performance index

$$
J_{s}=\int_{0}^{\infty}\left[p_{0}(x)+2 s_{0}^{\prime}(x) u_{0}+u_{0}^{\prime} R_{0}(x) u_{0}\right] \mathrm{d} t .
$$

It is assumed that the true model of (14)-(15) can be represented by a singularly perturbed system

$$
\begin{aligned}
\dot{x} & =a_{1}(x)+a_{2}(x) z, & & x \in R^{n} \\
\varepsilon \dot{z} & =a_{3}(x)+a_{4}(x) z+b(x) u, & & z \in R^{m}, \quad u \in R^{r}
\end{aligned}
$$

with the performance index

$$
J=\int_{0}^{\infty}\left[p(x)+s^{\prime}(x) z+z^{\prime} Q(x) z+u^{\prime} R(x) u\right] \mathrm{d} t,
$$

where $a_{1}, a_{2}, a_{3}, a_{4}^{-1}, b, p, Q$ and $R$ are continuously differentiable with respect to $x, p+s^{\prime} z+z^{\prime} Q z$ is a positive definite function of its arguments and $Q(x)$ and $R(x)$ are positive definite matrices for all $x$ of interest.

Our design philosophy is as follows. Let the set of equations (14)-(15) be the reduced slow subproblem representation of $(16)-(18)$ when $\varepsilon$ is formally set to zero (the full problem is well-posed). It is assumed that the effects of parasitic parameters are deteriorating the performance of the system. In other words, the design based on the reduced model satisfies the designer's objectives and requirements. Utilizing the concept of a slow manifold, we will show how a corrective slow control can be designed to achieve an exact slow optimal control for (16)-(18), in the sense that the optimal cost of the reduced system is equal to the optimal cost of the full system restricted to the manifold. To this end, let the manifold $M_{\varepsilon}$ and the slow control $u$ be expressed as

$$
\begin{aligned}
& z=h=h_{0}+\varepsilon h_{1}+\varepsilon^{2} h_{2}+\cdots \\
& u=u_{0}+\varepsilon u_{1}+\varepsilon^{2} u_{2}+\cdots .
\end{aligned}
$$

Substitution of (19)-(20) into (17) and equating like powers of $\varepsilon$ gives

$$
\begin{aligned}
& \varepsilon^{0}: a_{3}+a_{4} h_{0}+b u_{0}=0 \\
& \varepsilon^{1}: a_{4} h_{1}+b u_{1}=\dot{h}_{0} \\
& \varepsilon^{2}: a_{4} h_{2}+b u_{2}=\dot{h}_{1}
\end{aligned}
$$

and, in general

$$
\varepsilon^{k}: a_{4} h_{k}+b u_{k}=\dot{h}_{k-1}, \quad k \geq 1 .
$$

Further substitution of (19) into (16) leads to

$$
\dot{x}=a_{1}(x)+a_{2}(x)\left(h_{0}+\varepsilon h_{1}+\cdots\right) .
$$

At this stage a design specification is imposed on the manifold $M_{e}$, that is, we require $h=h_{0}$ and $h_{k}=0$ for $k=1,2, \ldots$. It turns out that this can be achieved successfully as follows. From (21), $h_{0}$ is given by

$$
h_{0}=-a_{4}^{-1}\left(a_{3}+b u_{0}\right) \text {. }
$$

To require $h_{1}=0$, and hence $M_{\varepsilon} M_{0}$, equation (22) should be satisfied for $u_{1}$ such that

$$
b u_{1}-\dot{h}_{0}=0 .
$$

The control $u_{1}$ is computable as long as (24) is a consistent equation, that is, rank $\{b\}=\operatorname{rank}\left\{B, \dot{h}_{0}\right\}$ for all $x$ of interest.

Remark. If there is no solution to $u_{1}$, then an exact optimal control cannot be achieved by just adding a single extra control to $u_{0}$, that is, we would have $\varphi_{1} \neq 0$ and $M_{\varepsilon} \neq M_{0}$. In this case we express the slow control as $u=u_{0}+\varepsilon u_{1}+$ $\varepsilon^{2} u_{2}+O\left(\varepsilon^{3}\right)$. Each extra control in $u$ will make the resulting performance index closer to the exact cost function. Note that the existence of an exact optimum control has already been discussed in Section 3. The power series expansion of the slow control is simply an approximate solution to this control.

With $u_{1}$ satisfying (24), we observe that $u_{k}=0$, $k=2,3, \ldots$, which results in $h_{k}=0, k=2,3, \ldots$ As a consequence, with $u=u_{0}+\varepsilon u_{1}$, the exact manifold $M_{\varepsilon}$ is now by design just the uncorrected manifold $M_{0}$. The reduced exact slow subsystem (23) becomes

$$
\dot{x}=a_{1}(x)+a_{2}(x) h_{0} .
$$

Deviation of the fast variable $z$ from $M_{0}$ denoted by $\eta$ satisfies the differential equation

$$
\varepsilon \dot{\eta}=a_{3}+a_{4}\left(\eta+h_{0}\right)+b u_{c}-\varepsilon \dot{h_{0}} .
$$

If we now write the composite control $u_{c}$ as

$$
u_{c}(x, \eta, \varepsilon)=u_{0}(x)+\varepsilon u_{1}(x)+u_{f}^{x}(\eta),
$$

where $u_{f}^{x}$ is a fast control, and substitute it in (25) and use (24), we get

$$
\varepsilon \dot{\eta}=a_{4}(x) \eta+b(x) u_{f}^{x}(\eta)-\varepsilon \frac{\partial h_{0}}{\partial x} a_{2}(x) \eta
$$

The fast subsystem is now obtained by letting $\varepsilon=0$, that is

$$
\frac{\mathrm{d} \eta}{\mathrm{d} \tau}=a_{4}(x) \eta+b(x) u_{f}^{x}(\eta)
$$

The fast subprobiem is to optimally control the fast subsystem (27) with respect to

$$
J_{f}=\int_{0}^{\infty}\left[\eta^{\prime} Q(x) \eta+\left(u_{f}^{x}\right)^{\prime} R(x)\left(u_{f}^{x}\right)\right] \mathrm{d} t .
$$

At this stage the reduced slow subproblem (14)-(15) and the reduced fast subproblem (27)-(28) are solved (Chow and Kokotovic, 1978a, b, 1981).

For $u_{c}(x, \eta, \varepsilon)$ to be a useful feedback control for the full system (16)-(18), it must be a stabilizing control. Utilizing the recently developed stability results of nonlinear singularly perturbed systems in Saberi and Khalil (1984) and Khorasani and Pai (1985), the stability properties of $(16)-(18)$ with $u_{c}$ given by (26) are next investigated.

The full system (16)-(18) is first written in the $x$ and $\eta$ state variables as

$$
\begin{aligned}
\dot{x} & =a_{0}(x)+b_{0}(x) u_{0}+a_{2}(x) \eta \\
\varepsilon \dot{\eta} & =\left(a_{4}(x)-\varepsilon \frac{\partial h_{0}}{\partial x} a_{2}(x)\right) \eta+b(x) u_{f}^{x}(\eta) .
\end{aligned}
$$

With $u_{0}(x)$ and $u_{f}^{x}(\eta)$ obtained from the reduced slow and fast subproblems (for details cf. Chow and Kokotovic, $1978 \mathrm{a}, \mathrm{b}, 1981)$, we rewrite (29)-(30) as

$$
\begin{aligned}
\dot{x} & =\bar{a}_{0}(x)+a_{2}(x) \eta \\
\varepsilon \dot{\eta} & =\left(\tilde{a}_{4}(x)-\varepsilon \frac{\partial h_{0}}{\partial x} a_{2}(x)\right) \eta
\end{aligned}
$$

where

$$
\begin{aligned}
& \bar{a}_{0}(x)=a_{0}(x)-b_{0}(x) R_{0}^{-1}(x)\left(s_{0}(x)+\frac{1}{2} b_{0}^{\prime}(x)\left(\frac{\partial V_{r}}{\partial x}\right)^{\prime}\right) \\
& \bar{a}_{4}(x)=a_{4}(x)-b(x) R^{-1}(x) b^{\prime}(x) K_{f}(x) .
\end{aligned}
$$

The reduced slow and fast subsystems are then obtained from $(31)-(32)$, as

$$
\begin{aligned}
\dot{x} & =\bar{a}_{0}(x) \\
d \eta / d \tau & =\bar{a}_{4}(x) \eta .
\end{aligned}
$$

We now assume that the following conditions are satisfied (Khorasani and Pai, 1985).

(a) The slow subsystem (33) has a positive definite Lyapunov function $V(x)$ such that

$$
\left[\nabla_{x} V(x)\right]^{\prime} \bar{a}_{0}(x) \leq-\alpha_{1} \bar{\Psi}^{2}(x), \quad \alpha_{1}>0
$$

where $\bar{\Psi}(x) \neq 0$ if $x \neq 0$ and $\dot{\Psi}(0)=0$. 
(b) The fast subsystem (34) has a positive definite Lyapunov function $W(\eta)$ such that

$$
\left[\nabla_{\eta} W(\eta)\right]^{\prime} \bar{a}_{4}(x) \eta \leq-\alpha_{2} \bar{\Phi}^{2}(\eta), \quad \alpha_{2}>0
$$

where $\bar{\Phi}(\eta) \neq 0$ if $\eta \neq 0$ and $\bar{\Phi}(0)=0$.

(c) $\left[\nabla_{X} V(x)\right]^{\prime} a_{2}(x) \eta \leq \alpha_{3} \vec{\Psi}(x) \Phi(\eta)+\alpha_{4} \tilde{\Phi}^{2}(\eta)$

(d) $\left[\nabla_{\eta} W(\eta)\right]^{\prime}\left(-\frac{\partial h_{0}}{\partial x} a_{2}(x) \eta\right) \leq \alpha_{5} \tilde{\Psi}(x) \Phi(\eta)+\alpha_{6} \tilde{\Phi}^{2}(\eta)$.

Conditions (c) and (d) establish the interconnection bounds between the slow and the fast subsystems.

Lemma 4.1. Assuming that conditions (a)-(d) hold, let $d \in(0,1)$ and $\varepsilon^{*}(d)$ be the positive number such that the matrix $T(d, \varepsilon)$ given by (35) is negative definite. Then for all $\varepsilon<\varepsilon^{*}(d)$ the origin $x=0, z=0$ is asymptotically stable and the function $U(x, \eta):=(1-d) V(x)+d W(\eta)$ is a Lyapunov function for $(31)-(32)$ guaranteeing this property.

Proof. (Outline.) It can be easily shown that the time derivative of $U(x, \eta)$ along the trajectories of (31)-(32) satisfies (Khorasani and Pai, 1985; Saberi and Khalil, 1984)

$$
\dot{U}(x, \eta) \leq\left[\begin{array}{ll}
\bar{\Psi}(x) & \bar{\Phi}(\eta)
\end{array}\right] T(d, \varepsilon)[\bar{\Psi}(x) \quad \bar{\Phi}(\eta)]^{\prime}
$$

where

$$
\begin{aligned}
& T(d, \varepsilon) \\
& =\left[\begin{array}{cc}
(d-1) \alpha_{1} & (1 / 2)\left[(1-d) \alpha_{3}+d \alpha_{5}\right] \\
(1 / 2)\left[(1-d) \alpha_{3}+d \alpha_{5}\right] & d\left(\alpha_{6}-\alpha_{2} / \varepsilon\right)+\alpha_{4}(1-d)
\end{array}\right] .
\end{aligned}
$$

The proof is now immediate.

The following lemma is a consequence of the above results.

Lemma 4.2. Let $u_{0}(x)$ be the minimizing control for the reduced slow subproblem $(14)-(15), u_{f}^{x}(\eta)$ be the minimizing control for the reduced fast subproblem $(27)-(28)$, and $u_{1}(x)$ be the corrective control designed to make the exact manifold $M_{\varepsilon}$ equal to $M_{0}$. Under these conditions there exists an $\varepsilon^{*}(d)>0$ such that the composite control $u_{c}$ in (26) is the exact optimal control strategy for $(16)-(18)$ for all $0 \leq \varepsilon<\varepsilon^{*}(d)$, in the sense that (i) $J=J_{s}$ on the manifold $M_{\varepsilon}$, (ii) $J=J_{s}+J_{f}+O(\varepsilon)$, and (iii) there exists a time $t_{1}>0$ such that $J=J_{s}$ for all $t>t_{1}$.

The asymptotic stability of an equilibrium point is not sufficient to guarantee the boundedness of an integral cost of the type (18) (for a counter example, refer to Chow and Kokotovic, 1981). It can be shown along the similar lines as in Chow and Kokotovic (1981) that the composite control $u_{c}$ given by (26) yields a bounded $J$ (details are omitted).

To illustrate the application of the above theory and to compare our improved results with those of Chow and Kokotovic (1978b) consider the problem of optimally controlling the system

$$
\begin{aligned}
\dot{x} & =x z \\
\varepsilon \dot{z} & =-z+u
\end{aligned}
$$

with respect to the performance index $J=\int_{0}^{x}\left(x^{4}+z^{2} / 2+\right.$ $\left.u^{2} / 2\right) \mathrm{d} t$. It can be shown that the reduced slow subproblem results in $u_{0}=-x^{2}$. The corrective control $u_{1}(x)$ is designed to achieve $M_{\varepsilon} \equiv M_{0}$. This gives from $(24), u_{1}(x)=2 x^{4}$. The overall slow control is therefore $u=-x^{2}+2 \varepsilon x^{4}$, and (31) becomes

$$
\dot{x}=-x^{3}+x \eta \text {, }
$$

where $\eta:=z+x^{2}$. Equation (30) with $u_{f}=-(\sqrt{ } 2-1) \eta$ (from the solution of the reduced fast subproblem) becomes

$$
\varepsilon \dot{\eta}=-\sqrt{ } 2 \eta+2 \varepsilon x^{2} \eta
$$

The reduced slow and fast subsystems constructed from (36) and (37), respectively, are given by

$$
\begin{aligned}
\dot{x} & =-x^{3} \\
\mathrm{~d} \eta / \mathrm{d} \tau & =-\sqrt{ } 2 \eta .
\end{aligned}
$$

The Lyapunov function for the slow subsystem is chosen as
$V(x)=x^{4} / 4$ and for the fast subsystem as $W(\eta)=\eta^{2} / 2$. In addition, let the comparison functions be $\bar{\Psi}(x)=x^{3}$ and $\bar{\Phi}(\eta)=\eta$. Conditions (a) and (b) are satisfied with $\alpha_{1}=1$ and $\alpha_{2}=1$. Conditions (c) and (d) result in the following inequalities, $x^{4} \eta-\alpha_{3} x^{2} \eta-\alpha_{4} \eta^{2} \leq 0$ and $2 \eta^{2} x^{2}-\alpha_{5} x^{3} \eta-$ $\alpha_{6} \eta^{2} \leq 0$, respectively. Let us assume that we specify the initial conditions of the system to be in the set $R=\{x, \eta|| x|\leq 0.8,| \eta \mid \leq 5\}$. With the choice of $\alpha_{i}=1$, $i=3, \ldots, 6$, inequalities (c) and (d) become

$$
R_{1}=\left\{x, \eta \mid x^{4} \eta-x^{2} \eta-\eta^{2} \leq 0\right\}
$$

and

$$
R_{2}=\left\{x, \eta \mid 2 \eta^{2} x^{2}-x^{3} \eta-\eta^{2} \leq 0\right\}
$$

respectively. The estimate of our region of attraction with the above choice of $\alpha$ s is given by $M=\{x, \eta \mid(x, \eta) \in$ $\left.\bigcap_{i=1}^{2} R_{i}, U(x, \eta)<c\right\}$ enclosed by $\bigcap_{i=1}^{2} R_{i}$, for some positive constant $c$. The upper bound on $\varepsilon$ is maximized by choosing $d=0.70$, which from (35) yields $\varepsilon^{*}(d)=0.382$. It is shown in Chow and Kokotovic (1978b) that for the initial conditions in $R$, the upper bound on $\varepsilon$ is $\varepsilon^{*}=0.03$. What have we shown here is that not only have we obtained a much less conservative bound on $\varepsilon$, furthermore we have $R \subseteq M$ and $J=J_{s}$ on $M_{\varepsilon}$.

\section{Conclusion}

The concept of a slow manifold is utilized to achieve an exact slow optimal control strategy for a general class of nonlinear singularly perturbed systems. The application to a class of nonlinear systems that are linear in the fast variable and control has been presented.

Acknowledgements - The author would like to acknowledge many fruitful discussions with Professors P. V. Kokotovic and $\mathrm{H}$. K. Khalil.

\section{References}

Chow, J. H. and P. V. Kokotovic (1976). A decomposition of near-optimum regulators for systems with slow and fast modes. IEEE Trans. Aut. Control, AC-21, 701-705.

Chow, J. H. and P. V. Kokotovic (1978a). Two-time scale feedback design of a class of nonlinear systems. IEEE Trans. Aut. Control, AC.23, 438-443.

Chow, J. H. and P. V. Kokotovic (1978b). Near-optimal feedback stabilization of a class of nonlinear singularly perturbed systems. SIAM J. Control Opt., 16, 756-770.

Chow, J. H. and P. V. Kokotovic (1981). A two-stage Lyapunov-Bellman feedback design of a class of nonlinear systems. IEEE Trans. Aut. Control, AC.26, 656-663.

Fenichel, N. (1979). Geometric singular perturbation theory for ordinary differential equations. $J$. Differential Equations, 31, 53-98.

Hoppensteadt, F. (1971). Properties of solutions of ordinary differential equations with small parameters. Communs Pure Appl. Math., 34, 809-840.

Khorasani, K. and M. A. Pai (1985). Asymptotic stability of nonlinear singularly perturbed systems using higher order corrections. Automatica, 21, 717-727

Kokotovic, P. V. (1984). Control theory in the 80s: trends in feedback design. Proc. 9th World Congress of IFAC, July 1984.

Kokotovic, P. V. and K. Khorasani (1984). Feedback control for nonlinear singularly perturbed systems: a geometric approach. Proc. JUREMA Symp. on Trends in Control Theory, July 1984, Cavtat, Yugoslavia.

Saberi, A. and H. Khalil (1984). Quadratic-type Lyapunov functions for singularly perturbed systems. IEEE Trans. Aut. Control, AC-29, 542-550.

Saberi, A. and H. Khalil (1985). Stabilization and regulation of nonlinear singularly perturbed systems-composite control, IEEE Trans. Aut. Control, AC-30, 739-747. 